AIP Applied Physics

\title{
High density InAIAs/GaAIAs quantum dots for non-linear optics in microcavities
}

R. Kuszelewicz, J.-M. Benoit, S. Barbay, A. Lemaître, G. Patriarche et al.

Citation: J. Appl. Phys. 111, 043107 (2012); doi: 10.1063/1.3682466

View online: http://dx.doi.org/10.1063/1.3682466

View Table of Contents: http://jap.aip.org/resource/1/JAPIAU/v111/i4

Published by the American Institute of Physics.

\section{Related Articles}

Quantitative strain mapping of InAs/InP quantum dots with $1 \mathrm{~nm}$ spatial resolution using dark field electron holography

Appl. Phys. Lett. 99, 261911 (2011)

Preparation of uncapped CdSe1-xSx semiconducting nanocrystals by mechanical alloying

J. Appl. Phys. 110, 124306 (2011)

Comparing retention and recombination of electrically injected carriers in Si quantum dots embedded in Si-rich SiNx films

Appl. Phys. Lett. 99, 243501 (2011)

Dilute-nitride GalnAsN/GaAs site-controlled pyramidal quantum dots

Appl. Phys. Lett. 99, 181113 (2011)

Dynamics of interatomic Coulombic decay in quantum dots

J. Chem. Phys. 135, 144112 (2011)

\section{Additional information on J. Appl. Phys.}

Journal Homepage: http://jap.aip.org/

Journal Information: http://jap.aip.org/about/about_the_journal

Top downloads: http://jap.aip.org/features/most_downloaded

Information for Authors: http://jap.aip.org/authors

\section{ADVERTISEMENT}

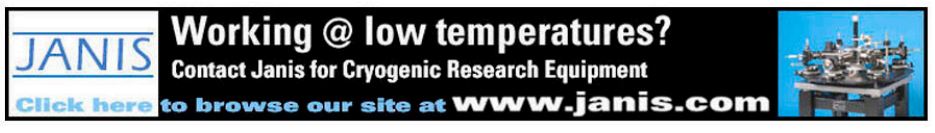




\title{
High density InAIAs/GaAIAs quantum dots for non-linear optics in microcavities
}

\author{
R. Kuszelewicz, ${ }^{1, a)}$ J.-M. Benoit, ${ }^{1}$ S. Barbay, ${ }^{1}$ A. Lemaître, ${ }^{1}$ G. Patriarche,${ }^{1}$ K. Meunier, ${ }^{1}$ \\ A. Tierno, ${ }^{2, b)}$ and T. Ackemann ${ }^{2, b)}$ \\ ${ }^{1}$ Laboratoire de Photonique et Nanostructures, Route de Nozay, Marcoussis 91460, France \\ ${ }^{2}$ University of Strathclyde, Glasgow, United Kingdom
}

(Received 25 July 2011; accepted 5 December 2011; published online 22 February 2012)

\begin{abstract}
Structural and optical properties of InAlAs/GaAlAs quantum dots grown by molecular beam epitaxy are studied using transmission electron microscopy and temperature- and time-resolved photoluminescence. The control of the recombination lifetime (50 ps-1.25 ns) and of the dot density $\left(5.10^{-8}-2.10^{11} \mathrm{~cm}^{-3}\right)$ strongly suggest that these material systems can find wide applications in opto-electronic devices as focusing non-linear dispersive materials as well as fast saturable absorbers. ㅇ 2012 American Institute of Physics. [doi:10.1063/1.3682466]
\end{abstract}

\section{INTRODUCTION}

In recent years, quantum dots (QD) have generated much interest as a new class of materials for non-linear and quantum optics. These synthetic structured materials exhibit unique features among semiconductor heterostructures, due to their atomic-like discrete states with energy transitions tunable through size and composition.

As an example, one of the most common experimental methods to elaborate self-assembled QD is molecular beam epitaxy (MBE) operated in the Stransky-Krastanov growth regime. The resulting QD sits on the so-called wetting layer (WL), consisting of a few-atomic-layer-thick quantum well. As a consequence, the three-dimensional confinement to which electrons are submitted generates a set of discrete energy levels whose spectral and dynamical properties are modified by their coupling to the WL continuum of states. The strength of this coupling depends on the potential barrier height via material composition of the QD. Nevertheless, the discrete character of the energy spectrum of QD provides optical properties that resemble those of an atomic system, with an access to both energy sides of electronic transitions. This property is of major importance, as an optical access to the high energy side of a transition allows for phenomena based on the focusing intensity-dependent index of refraction at excitation regimes below transparency. This property is never available in bulk and quantum well systems below transparency. ${ }^{1}$ Another property demonstrating the versatility of the QD material system is its possible use as a fast saturable absorber relying precisely on the interaction of discrete QD state electrons with the continuum of states in the WL. This latter mechanism is not the sole one that distinguishes ideal "atom-like" QD from real ones. Size fluctuation also brings up an inhomogeneous broadening, similar to that observable in atomic vapors, which selects specific classes in the interaction of QD with monochromatic light.

A theory of the QD susceptibility, which serves as a guideline all along this article, has been developed in Ref. 2,

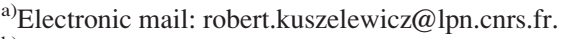

b) Present address: Institut Non Linéaire de Nice, 1361 route des Lucioles, Sophia Antipolis, Valbonne F-06560, France.
}

which incorporates the mechanisms described above and allows the description of optical nonlinear phenomena. Among these phenomena, we are particularly concerned with the temporal and spatial solitons relying on the focusing nonlinear dispersion, thereafter termed-though sometimes abusively-focusing Kerr effect (FKE). Cavity solitons (CS) are bistable localized optical states which can be independently written or erased at the input plane of large high Fresnel number resonators filled with a non-linear medium ${ }^{3,4}$ and which can be moved in the transverse plane of the resonator using amplitude or phase gradients of the field. ${ }^{5}$ Though some theoretical evidence has been made that self-defocusing media may, in very precise conditions, ${ }^{6}$ allow the existence of CS, it definitely appears that materials with focusing Kerr effect are most favorable to their existence with very little energy requirements. $^{7}$

QD have been developed first in the InAs/ $\mathrm{GaAs}^{8-11}$ system, which operates typically at wavelengths larger than $1 \mu \mathrm{m}$. The question of connecting with wavelength domains compatible with the GaAlAs material system and with Ti:Sa lasers is actually being raised. InAs QDs can obviously not provide the solution, since they lead to very shallow dots improper to the proposed target. Increasing the barrier height may be obtained by evenly incorporating aluminum in both the barrier and the dot materials, leading to a higher confinement of quantum states and to the forsaken spectral overlap with the GaAlAs system. In this framework, InAlAs/GaAlAs quantum dots open very promising perspectives, both for $\mathrm{cw}$ and pulsed nonlinear or even self-organizing optical systems. ${ }^{12,13}$ In this paper, structural and optical properties of MBE-grown InAlAs/GaAlAs quantum dots are investigated as a function of the growth kinetic and thermodynamical conditions. These conditions lead to controlling the density as well as the average size of QDs in a set of samples. However, this stage is also critical with respect to the amount of strain accumulated in the structure. This is why our study aims at determining the location and the density of eventual defects and correlating this with the growth conditions. This reveals an excellent approach for controlling the carrier lifetimes since the growth stage and introduces a means by which choosing between a slow FKE material $(\sim \mathrm{ns})$ and a fast (ps) 
saturable absorber becomes possible, as stated in Ref. 14. We also report on the measurement of nonlinear optical constants and, particularly, their saturation properties.

This article is organized as follows. In Sec. II, we describe the growth conditions of InAlAs/GaAlAs QD and correlate these to their structural characterization in terms of dot and dislocation densities. In Sec. III, spectral and temporal photoluminescence (PL) measurements are performed at low and room temperature. With the help of a phenomenological model inspired by Ref. 2, we analyze the dominant recombination mechanisms with respect to temperature. Sec. IV is dedicated to the measurement of the nonlinear optical properties and leads to useful evaluation of absorption coefficient and saturation intensity. Finally, the conclusion summarizes the main achievements of this paper and draws some perspectives.

\section{SAMPLE ELABORATION AND STRUCTURAL ANALYSIS}

The samples were MBE-grown on a GaAs substrate. Using the Stransky-Krastanov growth regime, a single plane of QDs was produced. As shown on Fig. 1, the structure consists of a $20 \mathrm{~nm} \mathrm{Ga}_{0.56} \mathrm{Al}_{0.44}$ As cladding layer followed by a $100 \mathrm{~nm} \mathrm{Al}_{0.28} \mathrm{Ga}_{0.72} \mathrm{As}$ barrier layer. The active region is composed of QDs sitting on a WL formed out of a few $\mathrm{Al}_{0.28} \mathrm{In}_{0.72} \mathrm{As}$ monolayers (ML). Three samples, $\mathrm{A}, \mathrm{B}$, and $\mathrm{C}$, were grown, containing, respectively, an initial deposition of InAlAs equivalent to $4.8 \mathrm{ML}, 5 \mathrm{ML}$, and 3.8 ML. The symmetric step-graded barrier and cladding layers with corresponding material concentration follow, and a GaAs cap terminates the structure. However, the kinetics were also adjusted so that the QD was grown in $4 \mathrm{~s}$ for $\mathrm{A}, 13.4 \mathrm{~s}$ for $\mathrm{B}$, and $12 \mathrm{~s}$ for $\mathrm{C}$. The growth duration allowing superficial matter diffusion controls the QD average size and, thereby, for their density. A fourth structure (D), comprising five QD layers and detailed in Sec. IV, was also grown. Each layer has characteristics very similar to those of sample C.

Transmission electron microscopy (TEM) cross-sectional and plane view images of samples $\mathrm{A}, \mathrm{B}$, and $\mathrm{C}$ were performed and are displayed in Fig. 2. The measurements resulting from these views confirm the values of the targeted thicknesses of the different layers, i.e., $100 \mathrm{~nm}$ and $20 \mathrm{~nm}$ for the barrier and cladding layers, respectively, except for sample A, where the bottom cladding layer is $10 \mathrm{~nm}$ only. The plane views give information on the QD density, which can

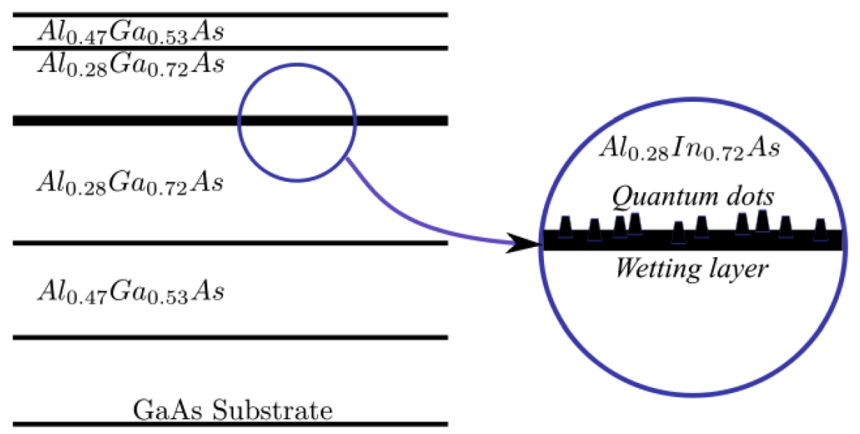

FIG. 1. (Color online) Generic scheme of structures.

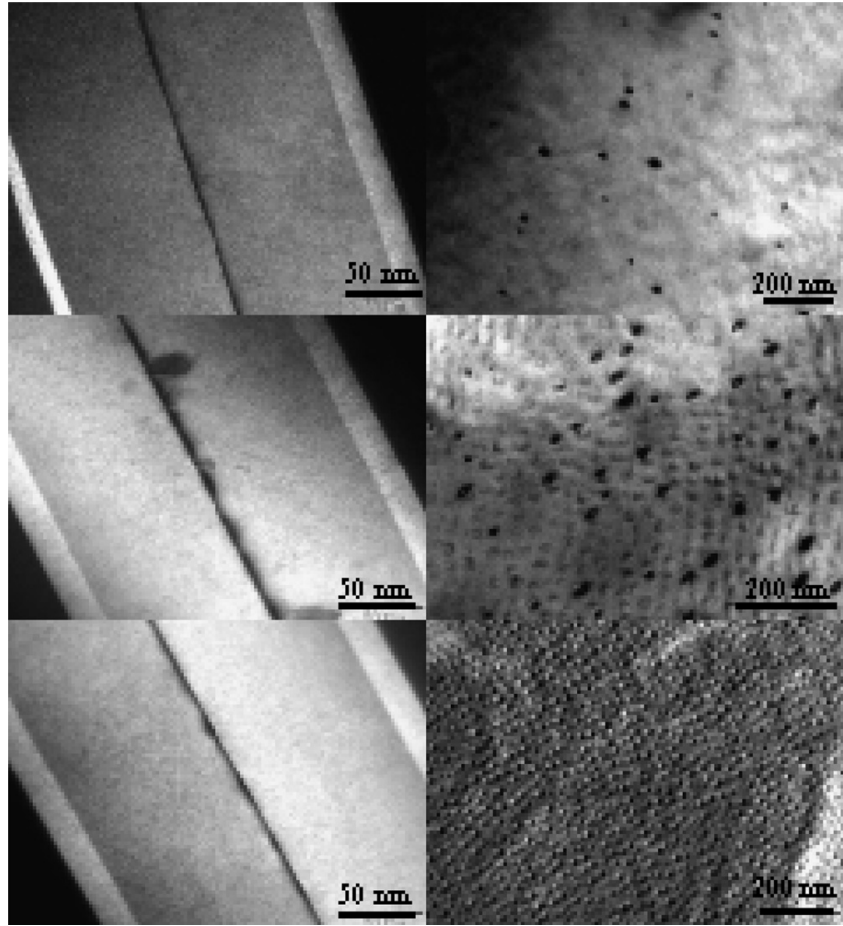

FIG. 2. TEM pictures of samples A, B, and C (from the top to the bottom), cross-section view (left), and plane view (right).

be estimated for the three samples as: $\rho_{A}=5.10^{8} \mathrm{~cm}^{-2}$, $\rho_{B}=5.10^{10} \mathrm{~cm}^{-2}$, and $\rho_{C}=2.10^{11} \mathrm{~cm}^{-2}$. These results are particularly promising, since theoretical simulations of QD susceptibility indicate that a density of the order of $10^{12} \mathrm{~cm}^{-2}$ is required for reaching the critical threshold for modulation instability in a resonator filled with a QD Kerr material. ${ }^{15} \mathrm{We}$ would like to emphasize that the density reached for sample $\mathrm{C}$ is very high and that it can hardly be increased without inducing the coalescence of the dots.

Looking at the sample B, cross-sectional view, it is also interesting to notice the dark contrasted zones originating from the QD or the WL and extending toward the barrier. This suggests a non-negligible amount of dislocations, either in the WL or at the WL-QDs/barrier layer interface.

Dislocations seem to occur as a relaxation mechanism of the strain accumulated by the deposition of multiple InAlAs MLs. In this respect, samples A, B, and C correspond to different numbers of ML, and sample B appears to be the most exposed to relaxation, with $5 \mathrm{ML}$ deposited. A key point lies in determining whether these dislocations concern the QD or the WL alone, as the TEM images suggest. This will be analyzed in Sec. III with PL measurements.

However, a trade-off must be reached between two conflicting targets, both involving the management of strain accumulation. On the one hand, the density of dots is controlled by the number of nucleation sites that increases with strain. On the other hand, the size of the dots depends on the quantity of matter deposited, on the ability for a nascent dot to drain the diffusing surrounding matter, or the tendency of neighboring dots to merge. This situation is directly controlled by the thermodynamical/kinetic conditions, under which the growth process is performed via the growth rate and the growth duration. 


\section{SPECTRAL CHARACTERIZATION}

Photoluminescence (PL) spectra have been obtained at room, intermediate, and low temperatures. In these spectra, one can identify the different layers according to the energy of the photons emitted, but also to the temperature dependence of the QD PL intensity. Room (300 K) and low (80 K) temperature spectra for samples $\mathrm{A}, \mathrm{B}$, and $\mathrm{C}$ are shown on the left side of Fig. 3.

For sample A at room temperature, four photoluminescence bands are observed that can be assigned, respectively, to the substrate $(1.42 \mathrm{eV})$, the QDs $(1.53 \mathrm{eV})$, the WL $(1.68 \mathrm{eV})$, and the barrier layer $(1.80 \mathrm{eV})$. At $80 \mathrm{~K}$, this sample displays three bands. The first one is attributed to the QDs $(1.70 \mathrm{eV})$, the second one, which appears as a shoulder on the first band, corresponds to the WL $(1.76 \mathrm{eV})$, and the last one, pointed at $1.85 \mathrm{eV}$, is related to the barrier layer. For this sample, we believe that the similarity of the two spectra at room and low temperature finds its origin in the low value of the QD density. Indeed, all PL bands are observed for both temperatures, suggesting that a small density of QD allows carrier trapping either in the WL or the barrier layer. However, carrier trapping in the QD seems to be enhanced at $80 \mathrm{~K}$, as the intensity of the PL band is higher than that of the WL, whatever the excitation power.

For sample B, only two PL bands are observed at any temperature for the GaAs substrate and the QD: at, respectively, $1.42 \mathrm{eV}$ and $1.49 \mathrm{eV}$ at room temperature and at
$1.51 \mathrm{eV}$ and $1.64 \mathrm{eV}$ at $80 \mathrm{~K}$. Here, we believe that the spectral bands associated with the WL and the barriers are not observed, most probably because high density dislocations act as non-radiative recombination centers and quench their luminescence.

For sample $\mathrm{C}$ at room temperature, we again observe four bands related to the substrate $(1.42 \mathrm{eV})$, the QDs $(1.63 \mathrm{eV})$, the WL $(1.74 \mathrm{eV})$, and the barrier layer $(1.84 \mathrm{eV})$. At $80 \mathrm{~K}$, the same sample displays only one band with a shoulder. The substrate and barrier layers are no longer observed, as we identify the peak at $1.77 \mathrm{eV}$ as being that of the QDs. The shoulder $(1.82 \mathrm{eV})$ of this band may be related to the WL luminescence. For sample $\mathrm{C}$, these results indicate that trapping of carriers in QDs at low temperature is more efficient than at room temperature. Let us note that, contrary to what happens with sample $\mathrm{A}$, at $80 \mathrm{~K}$, the barrier layer band is not observed anymore as a consequence of the higher QD density.

In order to extract more details on the recombination dynamics, the right side of Fig. 3 represents the variation of the QD integrated PL intensity as a function of the inverse of the temperature $1 / \mathrm{T}$. As one may expect for the three samples, the QD PL intensity decreases when increasing the temperature (dotted line). The straight line on each plot corresponds to an exponential fit with an activation energy law-type. The value extracted from the data yields an activation energy of around $100 \mathrm{meV}$, which is in excellent agreement with the energy shift measured between the QD and the WL. The
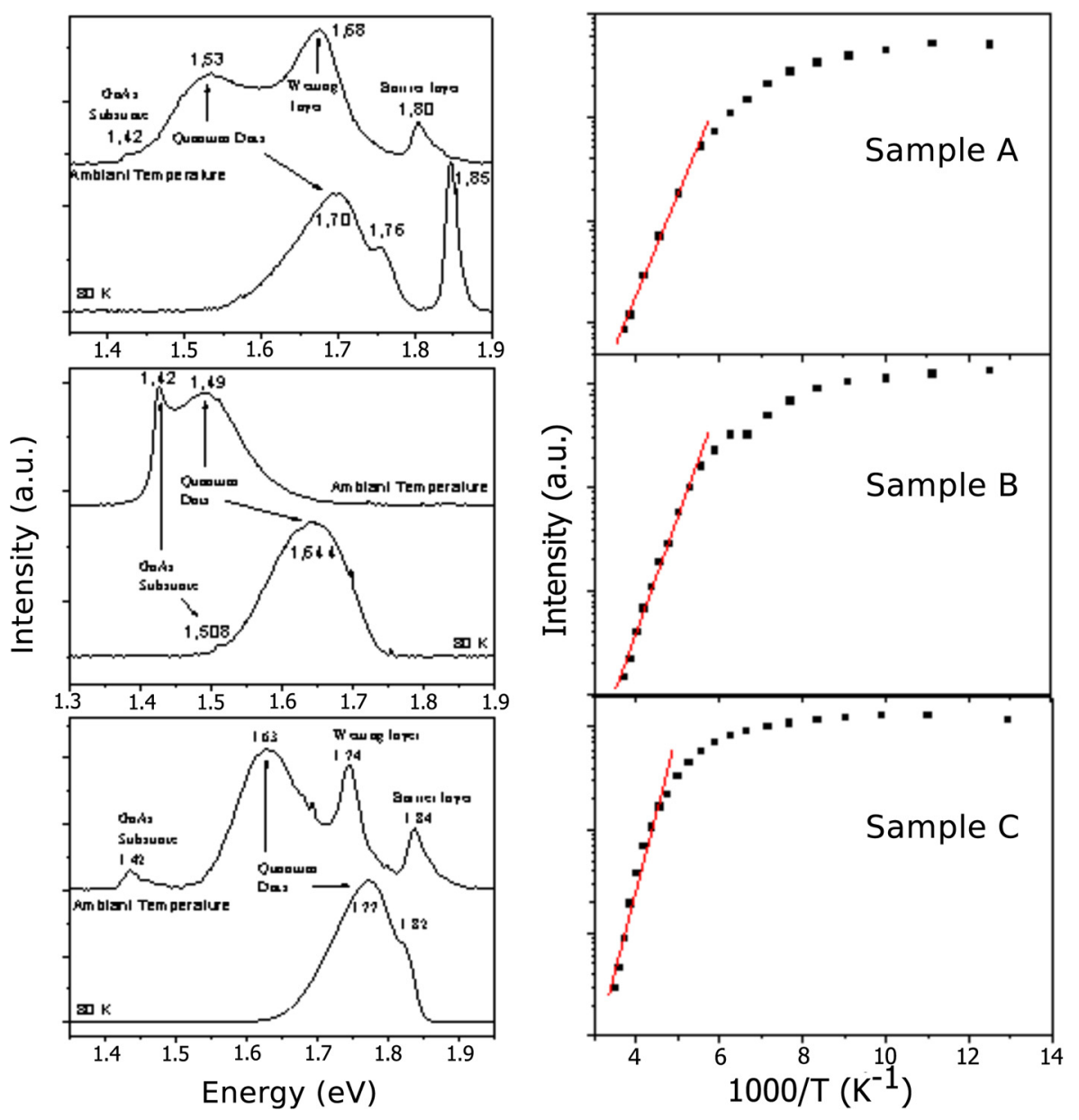

FIG. 3. (Color online) Luminescence spectra for samples A, B, and C (from top to bottom). Left: Room and low temperature. Right: Log of the normalized total PL intensity versus temperature. 
consistency of our results with an Arrhenius activation law not only suggests that thermal activation of carriers from the QD to the WL actually takes place in our samples, but also rules the recombination dynamics of the QD-WL system. To understand this behavior, we have used a rate equation model simplified with respect to our general model, ${ }^{2}$ which incorporates the thermal activation of the carriers from the QD to the WL, and that accurately describes the experimental PL collapse at the emission wavelength of QDs. In this case, the dynamical equations describing the carriers' densities in the WL $\left(N_{Q W}\right)$ and in the QD $\left(N_{Q D}\right)$, where the densities of electrons and holes are taken equal, read as

$$
\begin{gathered}
\frac{\partial N_{W L}}{\partial t}=I_{P}-\gamma_{W L} N_{W L}-\rho \sigma N_{W L}+\gamma_{T E} N_{Q D}, \\
\frac{\partial N_{Q D}}{\partial t}=\rho \sigma N_{W L}-\gamma_{Q D} N_{Q D}-\gamma_{T E} N_{Q D},
\end{gathered}
$$

where $I_{P}$ is the pump photon flux, $N_{W L}$ and $N_{Q D}$ are the respective WL and QD 2D carrier densities, $\gamma_{W L}$ and $\gamma_{Q D}$ are the WL and QD carrier recombination rates, $\rho$ is the $2 \mathrm{D}$ dot density, $\sigma$ is the single dot carrier capture cross section, and $\gamma_{T E}$ is the thermal activation rate from the QD to the WL with $\gamma_{T E}=\gamma_{0} \exp \left(-E / k_{b} T\right)$, where $E$ is the activation energy (barrier height). The diagram of the relevant mechanisms we accounted for is sketched in Fig. 4.

In the steady-state regime, through Eq. (1) and (2), we can determine $N_{Q D}$ as a function of $\gamma_{T E}$ as follows:

$$
N_{Q D}=\frac{\rho \sigma I_{P}}{\gamma_{Q D} \gamma_{W L}+\gamma_{Q D} \sigma \rho+\gamma_{T E} \gamma_{W L}} .
$$

Moreover, in a PL experiment, the QD luminescence intensity is given by

$$
I_{P L}=\gamma_{Q D}^{r a d} N_{Q D}
$$

where $I_{P L}$ is the QD emission intensity and $\gamma_{Q D}^{r a d}$ is the QD radiative recombination rate. In this model, we can adjust the parameters $E, \gamma_{0}$, and $\gamma_{W L}$, which indicates that dislocations should only affect the continuum states of the WL. In this representation, if the capture rate $\sigma$ is strong enough, the

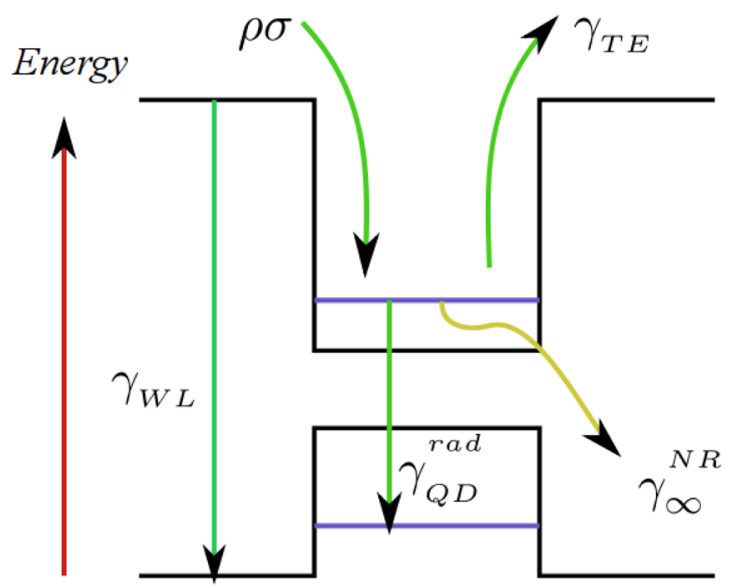

FIG. 4. (Color online) General schematics of the carrier excitation and relaxation channels in our QD model. overall lifetime should be governed by the escape rate from the QD.

According to this description, the dynamics of carriers and, thus, the PL intensity at the QD emission wavelength should be strongly dependent on the temperature range considered. At low temperature, with a strong capture rate, only very few carriers are able to escape and QD recombination rate prevails. At higher temperatures, a transition should occur at a temperature corresponding to the confinement energy $E$, above which thermal escape should, in turn, rule the recombination process. Sample A has a low density of QDs, which preserves its optical properties from being dominated by dislocations or defects. Experimental data and simulation of PL intensity measurements for this sample are presented in Fig. 5. The model allows us to determine the barrier height between the QD and the WL. The simulation actually leads to a value around $100 \mathrm{meV}$ for each sample, in agreement with the energy difference measured between the QDs and the WL.

The interpretation should also hold when the dislocations density is high, as in sample B, by accounting for the subsequent values of the recombination rates in the various parts of the sample. In the following, we proceeded to timeresolved photo-luminescence (TRPL) spectra measurements, and we selected two sets of samples, each set containing one piece of sample B and one piece of sample C. We then submitted one of these sets to hydrogenation in order to passivate the non-radiatively recombining centers. Hydrogenation was performed using RF-generated hydrogen plasma according to Ref. 16. The sample was thus maintained at a temperature of $200{ }^{\circ} \mathrm{C}$ in optimized conditions during $2 \mathrm{~h}$ under a hydrogen flux of $10 \mathrm{~cm}^{3} / \mathrm{min}$.

The non-radiative recombination rates of sample B (bottom of Fig. 6) and C (top of Fig. 6) dramatically decreases when increasing the temperature. However, in sample B, the lifetime is strongly ruled by the high density of defects (cf. middle of Fig. 2), leading to a maximum value of $450 \mathrm{ps}$ that is reached for a temperature around $200 \mathrm{~K}$, while the strong healing effect of hydrogenation restores a very high value of lifetime $(1250 \mathrm{ps}$ at $120 \mathrm{~K})$. On the contrary, hydrogenation on sample $\mathrm{C}$, which does not display any sizable amount of dislocations on TEM images (lower part of Fig. 2), leaves

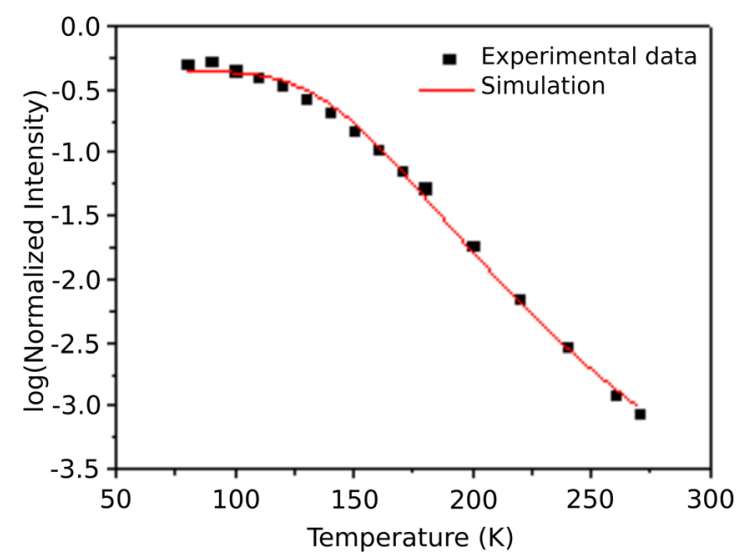

FIG. 5. (Color online) PL intensity at the QD emission wavelength: experimental data (squares) and simulation with the model developed in this paper (line) as a function of temperature for sample A. 


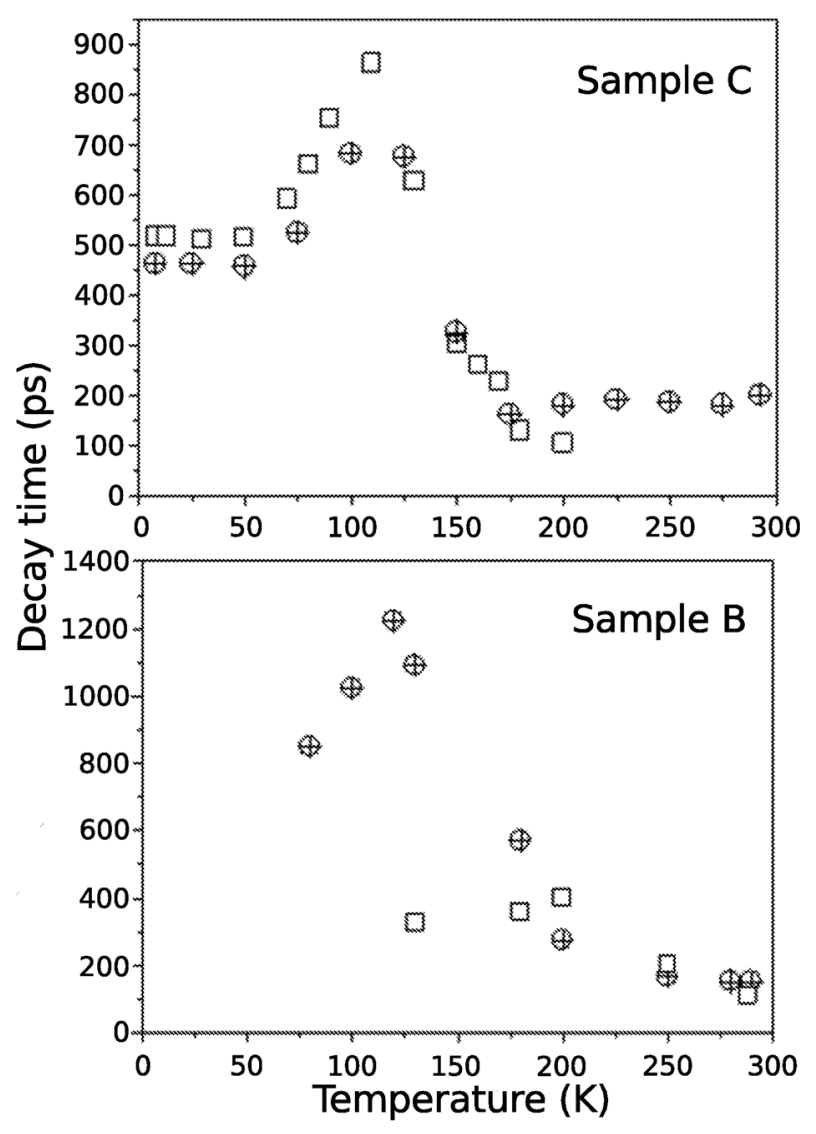

FIG. 6. Decay-time temperature dependence for raw (squares) and hydrogenated (cross) sample B (bottom) and C (top) obtained from time-resolved photoluminescence.

the recombination lifetime almost unaffected, while slightly decreasing from 900 ps to about 700 ps. The drastic changes observed in sample B between raw and hydrogenated pieces can be explained as follows: as hydrogenation passivates the non-radiative recombination center, the sensitivity to high temperature indicates that these centers strongly affect the QD state stability, as seen in the lower temperature range, while at higher temperature, when $k_{B} T$ reaches the barrier energy value, the thermal promotion of carrier occurs in both cases and PL dynamics is again ruled by the WL or barrier state dynamics. These results strongly assess the role of thermal activation of carriers in a QD material and attribute defects a direct influence on QD dynamics, even at low temperatures.

Finally, on the one hand, for sample $\mathrm{C}$ at room temperature, the recombination rate measured around 200 ps shows that we can consider using this material with a reasonable excitation power as a Kerr material. On the other hand, raw sample B displaying a recombination rate lower than $50 \mathrm{ps}$ at room temperature could be used as a fast saturable absorber for fast non-linear optical applications.

\section{OPTICAL MEASUREMENTS}

\section{A. Sample description}

In order to proceed to the measurements of the complex nonlinear index, an additional sample (sample D) was grown under the same thermodynamic and kinetic conditions as sample C. The motivation was the necessity to increase the volume of interaction between QDs and light by increasing the overall density of dots and, thereby, the sensitivity of our experimental setup to the QD contribution to the susceptibility. A solution in order to circumvent the conflicting requirements already mentioned in Sec. II between increasing the QD density and limiting the stress-induced defects was found in growing five superimposed planes of $\operatorname{In}_{0.67} \mathrm{Al}_{0.33} \mathrm{As}$ QDs with $\mathrm{Ga}_{0.67} \mathrm{Al}_{0.33} \mathrm{As}$ barriers (Fig. 7). A cross-sectional visualization of QDs was performed that gave information on the relaxation degree of the QDs. It was revealed comparable to that of sample $\mathrm{C}$ and, therefore, appropriate for optical experiments. Due to the depth at which QD layers are located from the surface, the density of dots $\rho_{D}$ could only be inferred from a cross-sectional TEM image and lead to an integrated density for the 5 layers, such that $2.10^{11}<\rho_{D}<5.10^{11} \mathrm{~cm}^{-2}$. Parameters are summarized in Table I. The complete structure was designed so as to form a planar waveguide inducing a vertical optical confinement. It comprises two $1.5-\mu$ m-thick $\mathrm{Ga}_{0.14} \mathrm{Al}_{0.86} \mathrm{As}$ cladding layers sandwiching the core layer, consisting of the five $3.8 \mathrm{ML} \operatorname{In}_{0.67} \mathrm{Al}_{0.33} \mathrm{As}$ QDs spaced by a 41.7-nm-thick $\mathrm{Ga}_{0.67} \mathrm{Al}_{0.33} \mathrm{As}$ barriers spacer. Finally, lateral optical confinement was introduced by etching the surface with a $0.4-\mu \mathrm{m}$-deep, 3- $\mu \mathrm{m}$-wide ridge. These values were retained on the basis of optical modeling with the finite element program ALCOR, warranting the monomode character of this waveguide around $800 \mathrm{~nm}$. The optical mode area at half-intensity is $3 \mu \mathrm{m} \times 0.250 \mu \mathrm{m}$.

\section{B. Theory}

The measurement technique relies on the use of a low finesse Fabry-Perot resonator consisting of a monomode waveguide operating around the transition energy of the QD. The $R=0.30$ reflectivity mirrors are the uncoated cleaved facets of the sample. We use the transmittance $T$ defined as the ratio of the transmitted $\left(I_{t}\right)$ to incident $\left(I_{i}\right)$ intensities of a lossless resonator,

$$
T=\frac{I_{t}}{I_{i}}=\frac{(1-R)^{2}}{(1-R)^{2}+4 R \sin ^{2}(\phi)},
$$

where $\phi$ is the phase accumulated during the propagation of light inside the cavity of length $L$. If it is filled with a material of intensity-dependent refractive index $n(I)$, the single pass phase accumulated is

$$
\phi \equiv \phi(I, L)=\int_{0}^{L} \frac{2 \pi n(I(z))}{\lambda} d z .
$$

In the presence of absorption with coefficient $\alpha$, one must use instead ${ }^{17,18}$

TABLE I. Parameters of the QD structures.

\begin{tabular}{lcccc}
\hline \hline & $\mathrm{A}$ & $\mathrm{B}$ & $\mathrm{C}$ & $\mathrm{D}$ \\
\hline Thickness (ML) & 4.8 & 5 & 3.8 & $5 \times 3.8$ \\
Time (s) & 4 & 13.6 & 12 & $5 \times 12$ \\
Density $\left(\mathrm{cm}^{-2}\right)$ & $5.10^{8}$ & $5.10^{10}$ & $2.10^{11}$ & $2-5.10^{11}$ \\
\hline \hline
\end{tabular}




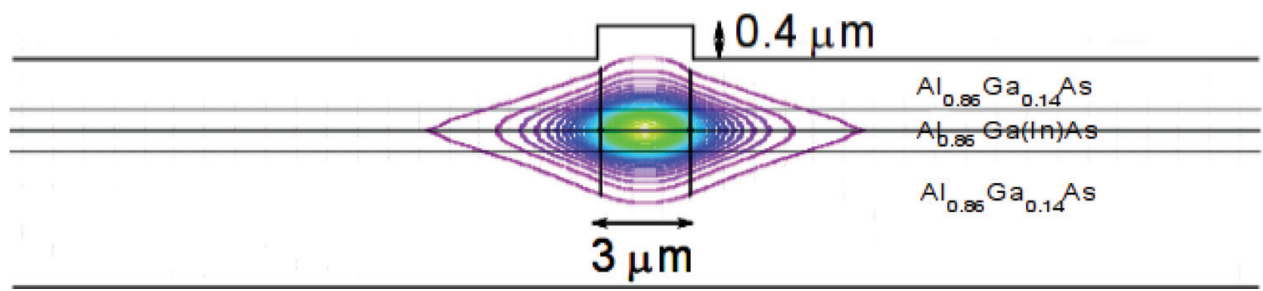

FIG. 7. (Color) 5-layer QD structure embedded in a ridge waveguide formed by the initial stack described in Fig. 1 modified for waveguiding properties. The single mode size calculated with a finite element program (ALCOR) is 3 $\mu \mathrm{m} \times 0.250 \mu \mathrm{m}$.

$$
T=\frac{(1-R)^{2} e^{-\alpha L}}{\left(1-R e^{-\alpha L}\right)^{2}+4 R e^{-\alpha L} \sin ^{2}(\phi)} .
$$

$\mathrm{T}$ is a function of the intracavity intensity $I$ via $\phi$ and $\alpha$. Similarly, the incident intensity $I_{i}$ can be connected to $I$ via $^{18}$

$$
\begin{aligned}
I_{i}= & \frac{\alpha L}{(1-R)\left(1+R e^{-\alpha L}\right)\left(1-e^{-\alpha L}\right)} \\
& \times\left[\left(1-R e^{-\alpha L}\right)^{2}+4 R e^{-\alpha L} \sin ^{2}(\phi)\right] I .
\end{aligned}
$$

The general resolution for arbitrary and eventually nonlinear $\phi$ 's may be found in solving Eq. (6) for $A=e^{-\alpha(I) L}$. Defining $S(I, \lambda) \equiv \sin ^{2}(\phi)$, this leads to the equation

$$
R^{2} T A^{2}+\left[2 R T(2 S-1)-(1-R)^{2}\right] A+T=0
$$

with the minus sign retained solution

$$
A=\frac{-\beta-\sqrt{\beta^{2}-4 R^{2} T^{2}}}{2 R^{2} T}
$$

and where $\beta(I, \lambda)$ is the coefficient of $A$ in the second order Eq. (8). This gives, in turn, an experimental access to the absorption coefficient coming straightforwardly as $\alpha=-\frac{\ln A}{L}$ and can be deduced from the knowledge of the transmission $T$. In turn, the nonlinear index determination is not straightforward and can only be accessed through the determination of the phase as an adjustable parameter. However, in the present case, the QDs are supposed to have a saturating $\left(\alpha_{0}\right)$ absorption law

$$
\alpha(I)=\alpha_{0} \frac{I_{s}}{I+I_{s}}
$$

and a saturating index

$$
n(I)=n_{\infty}-\delta n_{s} \frac{I_{s}}{I+I_{s}} .
$$

Equation (9) becomes an implicit equation in $A$, where the RHS depends on the transmission $T$ and on $A$ via $\beta$. This derivation of optical coefficients from experimental transmission measurements can be obtained via numerical or converging fit techniques.

\section{Principle of experiments}

The transmitted intensity $I_{t}$ is known to be proportional to the intracavity field $I$

$$
I_{t}=\frac{\alpha L e^{-\alpha L}(1-R)}{\left(1+R e^{-\alpha L}\right)\left(1-e^{-\alpha L}\right)} I .
$$

Under the effect of the real and imaginary parts of the nonlinear susceptibility, both the transmission spectrum-via $\phi-$ and the transmission amplitude maximum - via saturationare modulated. The combined measurement of $I_{t}$ and $I_{i}$ as a function of the wavenumber (wavelength) gives, thus, access to the transmittance and to the intracavity intensity. We neglect the eventual dependence of the intracavity intensity on the position and use instead the longitudinal average intensity $\bar{I}=\frac{1}{L} \int_{0}^{L} I(z) d z$. In the case of a waveguide, we use also the transverse confinement factor, which weighs the QD layer contribution to the nonlinear index according to the mode area. As a general formulation, $n(\bar{I}) \equiv n_{0}+\Gamma \delta n(\bar{I})$, the dependence on $\bar{I}$ should be linear or saturating. In the following, for the sake of simplification, we shall use $I$ instead of $\bar{I}$. The free spectral range $(F S R)$ corresponding to a phase shift $\Delta \phi=\pi$ under wavelength tuning and defined in wavenumber units as

$$
\Delta \sigma=\frac{1}{2 n_{0} L}
$$

appears to be affected by the intensity-dependent index of refraction and translates into the displacement of the $p$ th order resonance wavenumber $\sigma_{p}$ with $p=\phi(L) / \pi$ (Fig. 8). If a resonance is shifted by an amount $\delta \sigma_{\mathrm{p}}$, due to a nonlinear change $\delta n=\Gamma \delta n(I)$ of the refractive index, the stationarity of the phase at such a resonance yields

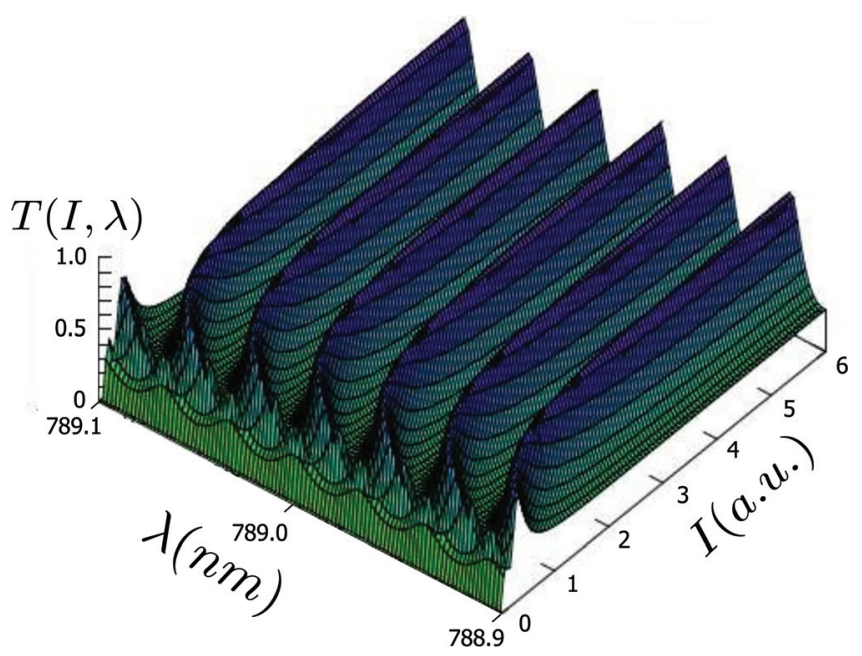

FIG. 8. (Color) Calculation of the transmission of nonlinear saturating Fabry-Perot resonator. The data used are: $R=0.3, \alpha_{0}=14 \mathrm{~cm}^{-1}, \alpha_{g}=10$ $\mathrm{cm}^{-1}, L=0.23 \mathrm{~cm}, I_{s}=2.8 \mathrm{~kW} / \mathrm{cm}^{2}, n_{0}=4.16, \delta n_{s}=1.75 \times 10^{-3}$. A confinement factor $=0.12$ is applied. 


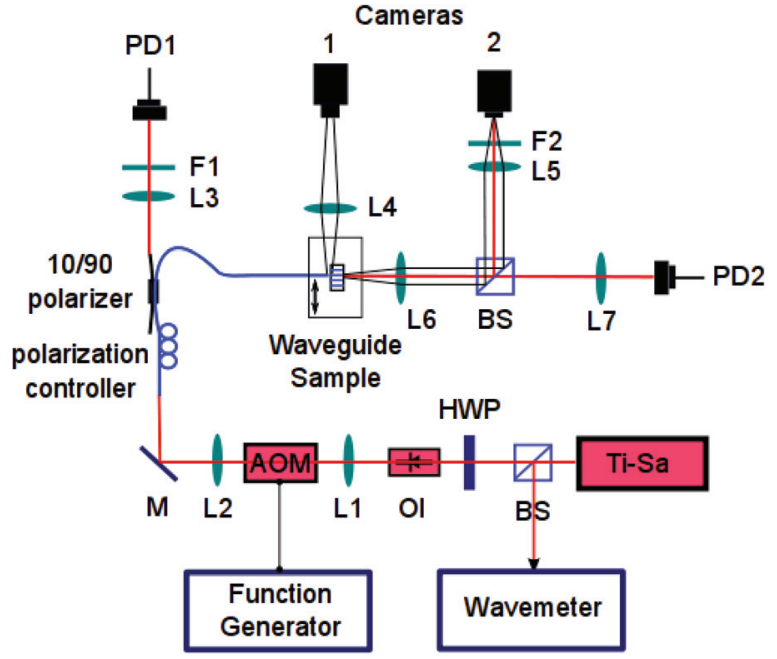

FIG. 9. (Color) Experimental setup: Ti:sapphire laser (Ti-Sa), beam splitter (BS), half-wave plate (HWP), optical isolator (OI), acousto-optic modulator (AOM), lenses L1 $(f=10 \mathrm{~mm}), \mathrm{L} 2(f=10 \mathrm{~mm}), \mathrm{L} 3 \quad(f=35 \mathrm{~mm}), \mathrm{L} 4$ $(f=50 \mathrm{~mm}), \mathrm{L} 5(f=35 \mathrm{~mm}), \mathrm{L} 6(f=35 \mathrm{~mm}), \mathrm{L} 7(f=35 \mathrm{~mm})$; mirror $(\mathrm{M})$, filters $(F 1, F 2)$, photodiodes (PD1, PD2).

$$
\delta n=-n \frac{\delta \sigma_{p}}{\sigma_{p}} .
$$

The 5 InAlAs QD layers have a total thickness of approximately $5 \times 6 \mathrm{~nm}=30 \mathrm{~nm}$. As they only partially overlap with the mode transverse dimension $250 \mathrm{~nm}$, we deduce a transverse confinement factor $\Gamma \simeq 0.12$. Bulk and MQW semiconductors are known to exhibit nonlinear index saturation of the order of $10^{-2}$. ${ }^{19}$ The experimental system must therefore be sensitive enough to detect a shift of the order of the FSR, i.e., $\delta \sigma_{\mathrm{p}} \approx \Delta \sigma$. Then one reads $\delta n \approx \frac{n}{p}$, showing that the sensitivity is amplified by a factor equal to the interference order, but reduced by the confinement factor $\Gamma$. This sensitivity can be reached if the cavity is long enough. For $L \approx 1 \mathrm{~mm}$, the interference order is typically $p \sim 10^{4}$ and the sensitivity $\Gamma \delta n \simeq 3-4 \cdot 10^{-4}$ for semiconductors.

\section{Experimental setup}

The experimental setup (Fig. 9) uses a Ti-Sa laser source operating in the short wavelength range between $760 \mathrm{~nm}$ and $810 \mathrm{~nm}$ and emitting $100-500 \mathrm{~mW} \mathrm{cw}$ power. A small fraction is collected via a beam splitter $(B S)$ into a Burleigh
WA1100 wavemeter. In order to prevent thermal drifts of the sample properties, the beam intensity is modulated by an acousto-optic modulator (AOM) in the deflection mode into rectangular or triangular envelopes with a $10 \mathrm{kHz}$ frequency and a duty cycle ranging from 10 to 20,converted from an electrical signal produced by an ANALOGIC 2000 arbitrary function generator. The modulated beam is then injected into a monomode fiber and directed to the monomode waveguide containing the QDs. In the path, light intensity is adjusted by a polarization controller injecting a 10/90 directional coupler, whose $10 \%$ branch allows the measurement of the incident intensity on photodiode PD1. The $90 \%$ branch is coupled via the lensed end of a tapered fiber into the waveguide, and its output, collected by a microscope objective (L6), is detected by a second photodiode PD2. The sample is mounted on a Peltier element holder giving a temperature control better than $0.1 \mathrm{~K}$. The alignment procedure is performed with the help of two cameras. Camera 1 allows the precise pre-positioning of the fiber with respect to the front facet of the waveguide, and Camera 2 allows the visualization of the rear facet and the optimization of the optical injection. Finally, 3 parameters could be measured: $I_{i}, I_{t}$, and $\lambda$. In practice, the measurement sequence consisted of ramping $I_{i}$ for a given $\lambda$ and measuring $I_{t}$, then deducing the intracavity intensity $I$ through Eq. (12), and finally recording and plotting the $3 D$ curve

$$
T \equiv T(I, \lambda)
$$

\section{E. Group index measurement}

A first series of measurements was performed in order to detect the nonlinear index variation under increasing illumination. The waveguide length is $L=0.23 \mathrm{~cm}$. Incident and transmitted intensities were recorded by ramping the incident intensity through the AOM at a fixed wavelength and iterating with $1 \mathrm{pm}$ wavelength steps over a $14 \mathrm{pm}$ range around the central wavelength. Such recordings were performed successively around $765 \mathrm{~nm}, 780 \mathrm{~nm}, 787 \mathrm{~nm}, 790 \mathrm{~nm}, 795 \mathrm{~nm}$, and $800 \mathrm{~nm}$ in order to scan different detunings from the inhomogeneously broadened line. Regular modulation are displayed at wavelengths around $\lambda=787 \mathrm{~nm}$ (left side of Fig. 10).

At low intensities, it was not possible to observe any drift of the Fabry-Perot resonances under increasing illumination, while at higher intensities, absorption saturation
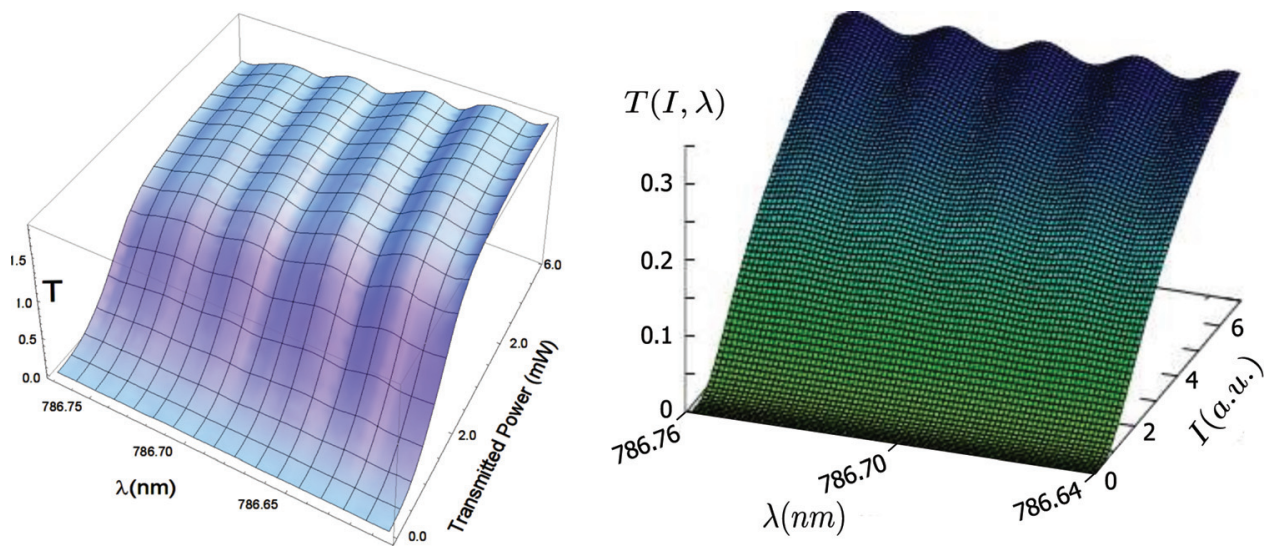

FIG. 10. (Color) Left: plot of the transmission as function of wavelength for different transmitted intensities. Right: transmission as a function of wavelength at constant intracavity intensity around $786.7 \mathrm{~nm}$. 


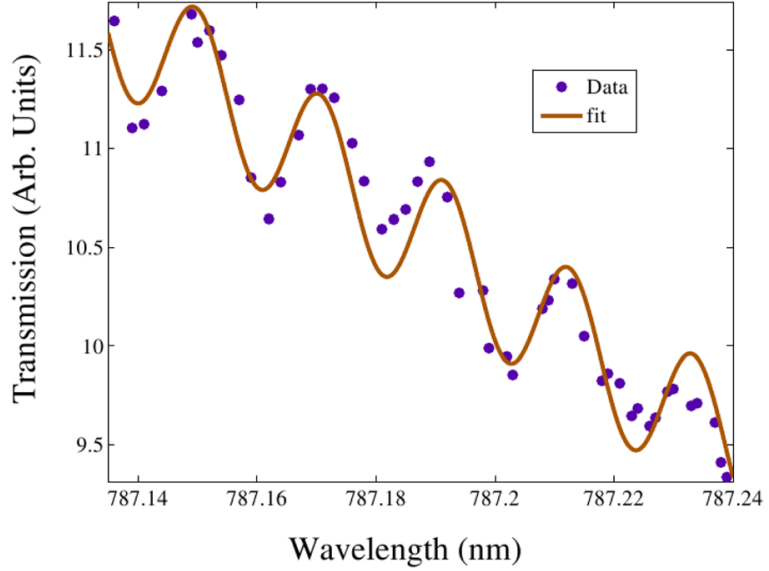

FIG. 11. (Color online) Transmission spectrum.

restores the phase modulation with a contrast poorer than expected. Both were attributed to the existence of a nonsaturable part of the absorption $\left(\alpha_{g}\right)$ that probably enforces the damping of the intracavity interferences at low intensities and maintains the cavity at a finesse lower than that of the cold cavity. This is confirmed by calculations (right side of Fig. 10) where absorption was modified as

$$
\alpha(I)=\alpha_{g}+\alpha_{0} \frac{I_{s}}{I+I_{s}} .
$$

From these data, a transmission spectrum at high intensity was extracted (Fig. 11), and a rather precise measurement of the free spectral range $\Delta \sigma=0.52 \mathrm{~cm}^{-1}$ was allowed to calculate the group index, incorporating the modal and spectral contributions to dispersion. This led to the group index value $n_{g}=4.16$.

\section{F. Absorption saturation}

The same experimental setup proved suitable to the measurement of the absorption of the quantum dots embedded in the waveguide section. The wavelength $\lambda=789.00 \mathrm{~nm}$ is chosen so as to lie rather close to the maximum of the QD absorption line. Transmission measurements, as expressed by Eq. (9), give, indeed, access to absorption. These were done, as previously, by ramping the incident intensity and measuring the transmittance. The right side of Fig. 12 shows the transmission as a function of the incident intensity. It is compared to theoretical curves deduced from Eqs. (12) and (7). The left side of Fig. 12 plots the absorption, as deduced from Eq. (9), applied to the experimental data and fitted with the ansatz of Eq. (10). The difficulty lies in the dependence of $T$ upon the nonlinear index via $\phi$. Therefore, instead of using the intracavity intensity, which depends on this phase term, we used the measured incident intensity with the assumption that they are in a quasi-constant ratio. We first adjusted the saturation parameters of the absorption in Eq. (12) so as to obtain the best possible fit. However, we noted that, in a second step, additional adjustments could be made by fine tuning the wavelength, i.e., the position with respect to the resonance and the saturation index. Further experiments will attempt to elucidate this point. However, from these adjusted values, we could extract the significant parameters of the QD material.

The calibration of the intracavity power was performed by using its relationship (Eq. (7)) with incident power $\left(P_{\max }^{\text {in }}=5.2 \mathrm{~mW}\right)$. In addition, we introduced the coupling coefficient of light into the waveguide $\gamma=0.083$ defined as the overlap ratio of the focused beam $3 \times 3 \mu \mathrm{m}^{2}$ to the mode area $S=0.250 \times 3 \mu \mathrm{m}^{2}$. The maximum intracavity power is, therefore, $P_{\max } \simeq 0.54 \mathrm{~mW}$.

The absorption curve appears, indeed, to have a saturation behavior leading to a vanishing absorption coefficient at high intensities. In the right side of Fig. 12 are presented two curves for $\alpha$ and for $1 /(\alpha)$ in the inset calculated with Eq. (9) and fitted according to a saturation ansatz also corrected with the transverse confinement factor $\Gamma$

$$
\alpha_{\text {measured }}(I)=\alpha_{g}+\Gamma \alpha_{0} \frac{P_{s}}{P+P_{s}},
$$
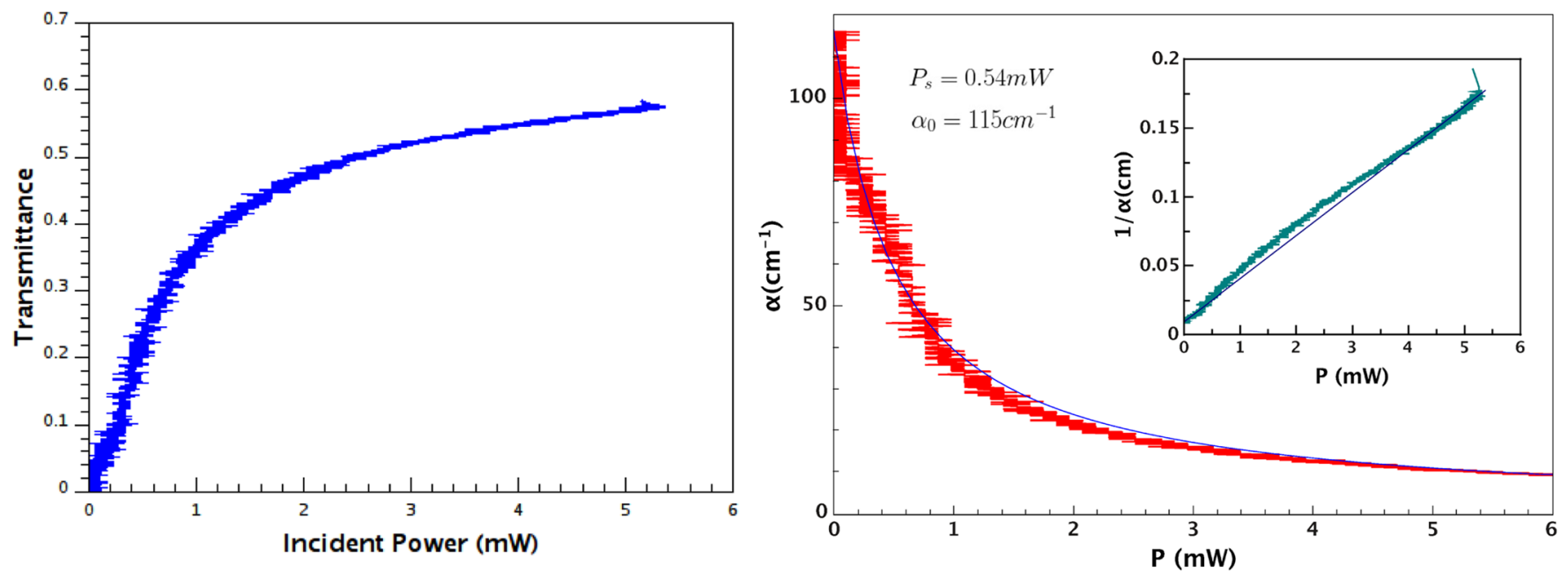

FIG. 12. (Color online) Left: plot of the transmission as a function of the incident intensity. Right: material absorption vs incident intensity as deduced from Eq. (9); thick line (experimental), thin line (theoretical). The inset displays the same data in the form $1 /=f\left(P_{\text {inc }}\right)$; the linear dependence assesses a saturation law of the absorption. 
where we used $\alpha_{g}=10 \mathrm{~cm}^{-1}$. They yield, with excellent fidelity, an unsaturated absorption value $\alpha_{0}=115 \mathrm{~cm}^{-1}$ and a saturation power $P_{s} \simeq 0.021 \mathrm{~mW}$. The deduced saturation intensity is

$$
I_{s}=\frac{P_{s}}{S}=2.8 \mathrm{~kW} / \mathrm{cm}^{2} .
$$

It could be interesting to compare this value to those obtained in other experiments. However, the comparison must be performed accounting with the experimental conditions at which they were performed, $\mathrm{cw}$ or pulsed. In the $\mathrm{cw}$ regime, gain and absorption measurements have been performed recently by some of the authors on $1.26 \mu \mathrm{m}$ InAs/GaAs edge emitter lasers, leading to saturation or gain intensities of the order of $10^{9} \mathrm{~W} / \mathrm{m}^{2}$ under various electrical injection around transparency. ${ }^{20}$ In the pulsed regime, different characteristic times have to be considered (pulse duration or recombination lifetime). ${ }^{21}$ In the $\mathrm{cw}$ regime, QDs are expected, in general, to have a lower saturation intensity than material of lower confinement dimensionality. In Ref. 19, for example, a series of measurements were performed on bulk and MQW Urbach's tails, showing that the relevant parameter for comparison is the factor $\alpha_{0} I_{s}$ proportional to the transparency carrier density. We obtain here $\alpha_{0} I_{s} \simeq 3.10^{5} \mathrm{~W} . \mathrm{cm}^{-3}$, which confirm the reduction of the saturation parameters in InAlAs/GaAlAs QDs, whose values were, respectively, in the range 0.1$1 \times 10^{8}$ W.cm ${ }^{-3}$ for bulk GaAs and AlGaAs/GaAs multiquantum wells. In parallel, the saturation index was of the order of $\delta n_{s} \simeq 2-3 \times 10^{-2}$. These comparisons are only qualitative and would require a specific study including the dot density as a parameter.

\section{CONCLUSION}

In this paper, we reported the realization and characterization of InAlAs/GaAlAs QDs layers produced by MBE technique in the Stransky-Krastanov growth regime. Their structural characteristics were analyzed with TEM and allowed to assess the possibility to control the QD density via the growth conditions. Spectral and dynamical properties were also measured by photoluminescence experiments, which allowed us to evaluate the confinement energy of carriers and the transition temperature for the onset of thermoemission processes.

Finally, we realized a device in the form of a waveguide creating the conditions of measuring the linear and nonlinear optical coefficients of InAlAs/GaAlAs quantum dots. The group index could be evaluated as $n=4.16$ and the saturation of the absorption assessed. This phenomenon could be associated to a value of the unsaturated absorption coefficient $\alpha_{0} \simeq 115 \mathrm{~cm}^{-1}$, a saturable contribution to the refractive index $\delta n_{s} \simeq 1.4 \times 10^{-3}$, and finally to a saturation intensity of $I_{S} \simeq 2.8 \mathrm{~kW} / \mathrm{cm}^{-2}$.

Further experiments associated to the model recalled here should give access to the value of the nonlinear index and confirm the interest of such a material, both for the use as a nonlinear cavity material for optical self-organization and for a saturable absorber in active systems.

${ }^{1}$ W. W. Chow, S. W. Koch, and M. Sargent III, Semiconductor-Laser Physics (Springer-Verlag, Berlin, 1994).

${ }^{2}$ I. M. Perrini, S. Barbay, T. Maggipinto, M. Brambilla, and R. Kuszelewicz, Appl. Phys. B 81, 905 (2005).

${ }^{3}$ S. Barland, J. Tredicce, M. Brambilla, L. Lugiato, S. Balle, M. Giudici, T. Maggipinto, L. Spinelli, G. Tissoni, T. Knoedel, M. Miller, and R. Jaeger, Nature 419, 699 (2002).

${ }^{4}$ S. Barbay, Y. Ménesguen, X. Hachair, L. Leroy, I. Sagnes, and R. Kuszelewicz, Opt. Lett. 31, 1504 (2006).

${ }^{5}$ W. Firth and A. Scroggie, Phys. Rev. Lett. 76, 1623 (1996).

${ }^{6}$ M. Brambilla, L. Lugiato, and M. Stefani, Electron. Lett. 34, 109 (1996).

${ }^{7}$ L. Lugiato and R. Lefever, Phys. Rev. Lett. 21, 2209 (1987).

${ }^{8}$ J.-M. Gérard and J.-Y. Marzin, Appl. Phys. Lett. 53, 568 (1988).

${ }^{9}$ D. Leonard, M. Krishnamurthy, C. M. Reaves, S. P. Denbaars, and P. M. Petroff, Appl. Phys. Lett. 63, 3203 (1993).

${ }^{10}$ J. M. Moison, F. Houzay, F. Barthe, L. Leprince, E. André, and O. Vatel, Appl. Phys. Lett 64, 196 (1994).

${ }^{11}$ J. Gérard, J. Génin, J. Lefebvre, J. Moison, N. Lebouché, and F. Barthe, J. Cryst. Growth 150, 351 (1995).

${ }^{12}$ R. Leon, S. Fafard, D. Leonard, J. Merz, and P. Petroff, Appl. Phys. Lett. 67, 521 (1995).

${ }^{13}$ K. Hinzer, P. Hawrylak, M. Korkusinski, S. Fafard, M. Bayer, O. Stern, A. Gorbunov, and A. Forchel, Phys. Rev. B 63, 075314 (2001).

${ }^{14}$ J. Pulka, T. Piwonski, G. Huyet, J. Houlihan, S. Barbay, A. Martinez, K. Merghem, A. Lemaître, A. Ramdane, and R. Kuszelewicz, IEEE J. Quantum Electron. QE47, 1094 (2011).

${ }^{15}$ S. Barbay, J. Koehler, R. Kuszelewicz, T. Maggipinto, I. Perrini, and M. Brambilla, IEEE J. Quantum Electron. 39, 245 (2003).

${ }^{16}$ O. Chen, P. Chu, T. Höchbacher, J.-K. Lee, M. Nastasi, D. Buca, S. Manti, R. Loo, M. Caymax, T. Alford, J. Mayer, N. David Theodore, M. Cai, B. Schmidt, and S. S. Lau, Appl. Phys. Lett. 86, 031904 (2005).

${ }^{17}$ D. Miller, IEEE J. Quantum Electron. QE-17, 306 (1981).

${ }^{18}$ B. Wherrett, IEEE J. of Quantum Electron. QE-20, 646 (1984).

${ }^{19}$ B. G. Sfez, J. L. Oudar, R. Kuszelewicz, and D. Pellat, Appl. Phys. Lett. 80, 1163 (1992).

${ }^{20}$ A. Tierno, T. Ackemann, C. Leburn, and C. A. Brown, Appl. Phys. Lett. 97, 231104 (2010).

${ }^{21}$ A. Tierno, T. Ackemann, T. Maggipinto, and M. Brambilla, Phys. Rev. B 80, 035314 (2009). 


\section{Erratum: "High density InAIAs/GaAIAs quantum dots for non-linear optics in microcavities" [J. Appl. Phys. 111, 043107 (2012)]}

R. Kuszelewicz, ${ }^{1}$ J.-M. Benoit, ${ }^{1}$ S. Barbay, ${ }^{1}$ A. Lemaître, ${ }^{1}$ G. Patriarche,${ }^{1}$ K. Meunier, ${ }^{1}$ A. Tierno, ${ }^{2}$ and T. Ackemann ${ }^{2}$

${ }^{1}$ Laboratoire de Photonique et Nanostructures, Route de Nozay, Marcoussis 91460, France

${ }^{2}$ University of Strathclyde, Glasgow, United Kingdom

(Received 29 April 2012; accepted 11 May 2012; published online 29 June 2012)

[http://dx.doi.org/10.1063/1.4731632]

Text of Acknowledgment to be added after the conclusion section: ${ }^{1}$

\section{ACKNOWLEDGMENTS}

T. Ackemann and A. Tierno gratefully acknowledge support by EPSRC project EP/E025021, A. Tierno also acknowledges a travel grant from COST action MP0702.

${ }^{1}$ R. Kuszelewicz, J.-M. Benoit, S. Barbay, A. Lemaître, G. Patriarche, K. Meunier, A. Tierno, and T. Ackemann, J. Appl. Phys. 111, 043107 (2012). 